\title{
Pheochromocytomas, MEN 2A and Pregnancy: A Case Report with Review of the Literature
}

\author{
Benzon M Dy, Eitan Podgaetz, William F Young, Geoffrey B Thompson
}

\begin{abstract}
Pheochromocytoma in pregnancy is a rare condition with an estimated incidence of one in 54,000 pregnancies. Pheochromocytoma in the setting of multiple endocrine neoplasia (MEN) type $2 \mathrm{~A}$ develops in approximately $40 \%$ of the patients with this germline mutation. MEN 2A patients with pheochromocytoma are often asymptomatic compared to those with nonsyndromic pheochromocytoma. We present a 28-year-old pregnant woman with a known MEN 2A mutation who was incidentally found to have a pheochromocytoma. She underwent an uncomplicated laparoscopic right adrenalectomy after proper $\alpha$ - and $\beta$-adrenergic blockade. Her serum fractionated metanephrines normalized prior to discharge from the hospital. The remainder of her pregnancy was uneventful and she delivered a health baby at term. Pheochromocytoma in pregnancy requires careful management to assure the well being of both the mother and the fetus. In pregnancy, laparoscopic surgical removal of the pheochromocytoma is feasible with excellent results when performed in the second trimester. In late pregnancy, medical management with close monitoring of the mother and fetus is recommended until fetal maturity is achieved. In the third trimester, cesarean section followed by open adrenalectomy is the preferred operation. An integrated and multidisciplinary approach with close communication among the endocrinologist, surgeon and perinatal services is paramount to achieve excellent outcomes for both patients. Women with MEN 2 should be advised to refrain from pregnancy until updated biochemical and imaging studies have ruled out pheochromocytoma.
\end{abstract}

Keywords: MEN 2A, Pheochromocytoma, Pregnancy.

How to cite this article: Dy BM, Podgaetz E, Young WF, Thompson GB. Pheochromocytomas, MEN 2A and Pregnancy: A Case Report with Review of the Literature. World J Endocr Surg 2012;4(1):29-32.

\section{Source of support: Nil}

Conflict of interest: None

\section{INTRODUCTION}

A 28-year-old asymptomatic woman was referred by her local primary care physician for definitive management of her pheochromocytoma at 10 weeks gestation. Physical examination revealed a blood pressure of 100/71 mm Hg, pulse of 77 beats per minute, normal heart and lung findings.

She had been diagnosed with a RET proto-oncogene mutation at age 14 and underwent a total thyroidectomy with no evidence of medullary thyroid cancer. She had annual biochemical testing for pheochromocytoma, testing positive for the first time in 2006 at age 26 and a $2 \mathrm{~cm}$ left adrenal mass was identified on imaging. She underwent laparoscopic left adrenalectomy at an outside hospital without complications. Her CT scan at that time did not reveal an abnormality in the right adrenal gland (Fig. 1).

After her left adrenalectomy, she had been attempting to become pregnant and had miscarriages in September of 2007 and January of 2008.

A follow-up CT scan of her abdomen in February of 2008 revealed a $2.8 \times 1.9$ right adrenal mass (Fig. 2).

Her laboratory values are mentioned in Table 1.

The $\beta$-hCG screening test was positive, confirmed with a quantitative $\beta$-hCG and a fetal ultrasound.

She had been relatively asymptomatic with systolic pressures of 100 to $110 \mathrm{~mm}$ Hg since her left adrenalectomy 2 years previously. However, over those 2 years she had experienced occasional palpitations and a sense of a 'rush' when she laid down.

The patient was started on an $\alpha-1$ adrenergic receptor antagonist, doxazosin $1 \mathrm{mg}$ by mouth daily at bedtime, and she continued to monitor her blood pressure twice daily. Two weeks prior to her planned right adrenalectomy, doxazosin was discontinued and phenoxybenzamine was initiated at $10 \mathrm{mg}$ daily. Five days before her procedure, she was started on propranolol $10 \mathrm{mg}$ four times daily for the first 2 days and then switched to long acting propranolol $60 \mathrm{mg}$ daily. The phenoxybenzamine dosage was increased to $20 \mathrm{mg}$ per day 3 days prior to her surgery.

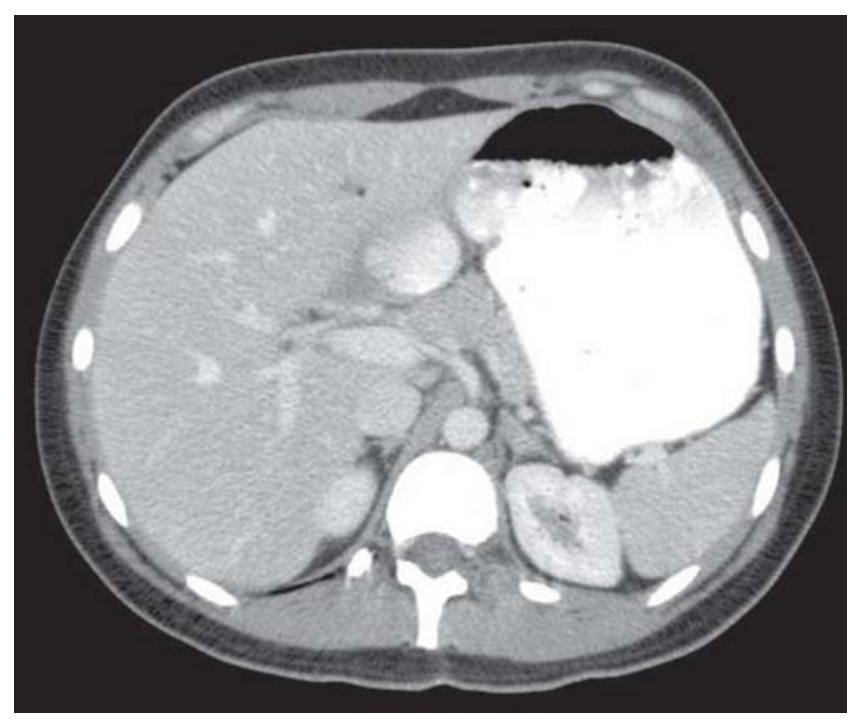

Fig. 1: Normal right adrenal gland in 2005 during evaluation for her left adrenal pheochromocytoma 
She also met with our perinatal specialists before her surgery who recommended pre- and postprocedure fetal heart rate assessments, epidural analgesia and serial ultrasound every 3 to 4 weeks until completion of her pregnancy. Because of her previous adrenalectomy, resection of her pheochromocytoma with an adrenalectomy would require permanent glucocorticoid and mineralocorticoid replacement therapy.

The morning of her procedure, she received methylprednisolone sodium succinate $20 \mathrm{mg}$ intravenously and another $20 \mathrm{mg}$ that same evening. She then underwent an uneventful laparoscopic right adrenalectomy. There where no intraoperative blood pressure abnormalities during the operation. Blood loss was minimal. Pathology revealed a $3.0 \times 3.0 \times 2.8 \mathrm{~cm}$ pheochromocytoma. Her glucocorticoid dosages were tapered to a dismissal dosage of hydrocortisone $10 \mathrm{mg}$ twice daily and fludrocortisone $0.1 \mathrm{mg}$ daily.

Fetal heart sounds pre- and postoperatively were monitored and normal (Fig. 3).

She recovered well and was discharged home 2 days later in good condition. Ten days after her procedure, she was feeling well and her plasma metanephrines and normetanephrines normalized.

The patient was advised to continue annual fractionated metanephrine, calcitonin and serum calcium testing.

\section{PHEOCHROMOCYTOMA, PREGNANCY AND MEN 2A}

Pheochromocytoma during pregnancy is a rare phenomenon occurring in fewer than $0.002 \%$ of all pregnancies. ${ }^{1}$

Pheochromocytomas arise from the chromaffin and sustentacular cells of the adrenal medulla and in extraadrenal paraganglia.

As many as one fourth of pheochromocytomas can be associated with the RET proto-oncogene mutations (MEN

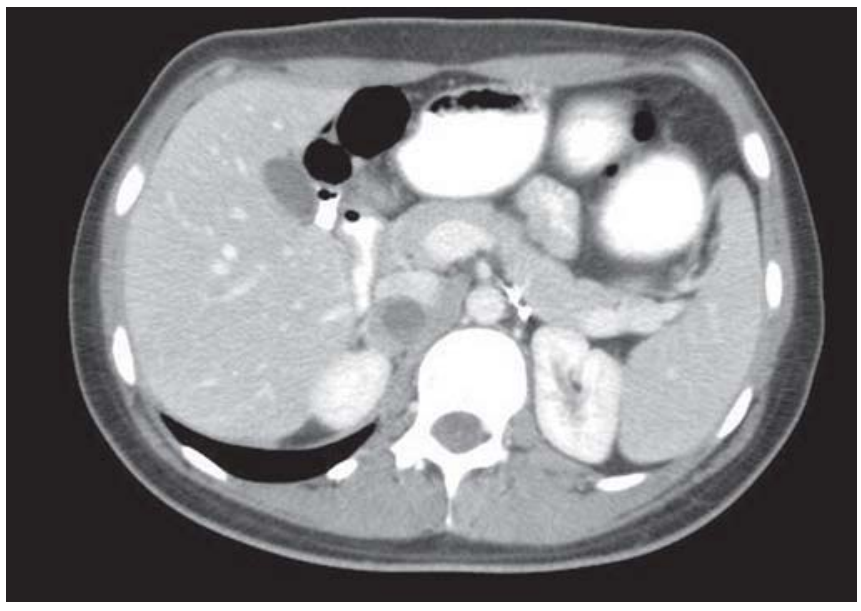

Fig. 2: CT scan in 2008: Right adrenal mass measuring $2.8 \times 1.9 \mathrm{~cm}$
2A and 2B), the VHL tumor suppressor gene mutations (Von Hippel-Lindau disease) and with NF1 tumor suppressor gene mutations (neurofibromatosis type 1 ). With modern molecular analysis, the familial incidence of pheochromocytoma and paraganglioma has proven to be approximately $25 \% .^{2,3}$

MEN 2 is an autosomal dominant disorder with an estimated prevalence of 2.5 per 100,000. MEN 2A has a hereditary predisposition to medullary thyroid carcinoma (90\%), pheochromocytoma (40-50\%) and primary parathyroid hyperplasia (10-20\%). Pheochromocytomas in MEN 2A patients are bilateral in $40 \%$ of patients as opposed to sporadic disease, which is most often unilateral. ${ }^{4}$ Germline mutation testing is the best screening test for this disorder.

Classically, pheochromocytomas secrete epinephrine, norepinephrine or dopamine, but a milieu of other hormones have been isolated from these tumors. Patients may either be asymptomatic or present with several hallmarks of pheochromocytomas, such as hypertensive spells with headaches, sweating, palpitations, paroxysmal hypertension or feelings of impending 'doom'.

Pheochromocytoma in pregnancy carries a maternal mortality rate of approximately $40 \%$ and a fetal death rate exceeding $50 \% .^{5}$

\begin{tabular}{lll}
\hline \multicolumn{3}{c}{ Table 1: Laboratory values } \\
\hline Measurement & $\begin{array}{c}\text { Reference } \\
\text { range }\end{array}$ \\
\hline Urine metanephrine & $521 \mu \mathrm{g} / 24$ hour & $<400 \mu \mathrm{g} / 24$ hour \\
Urine normetanephrine & $678 \mu \mathrm{g} / 24$ hour & $<900 \mu \mathrm{g} / 24$ hour \\
Urine norepinephrine & $60 \mu \mathrm{g} / 24$ hour & $<20 \mu \mathrm{g} / 24$ hour \\
Urine epinephrine & $15 \mu \mathrm{g} / 24$ hour & $<20 \mu \mathrm{g} / 24$ hour \\
Plasma metanephrine & $0.59 \mathrm{nmol} / /$ & $<0.5 \mathrm{nmol} / /$ \\
Plasma normetanephrine & $2.26 \mathrm{nmol} / /$ & $<0.9 \mathrm{nmol} / \mathrm{l}$ \\
\hline
\end{tabular}

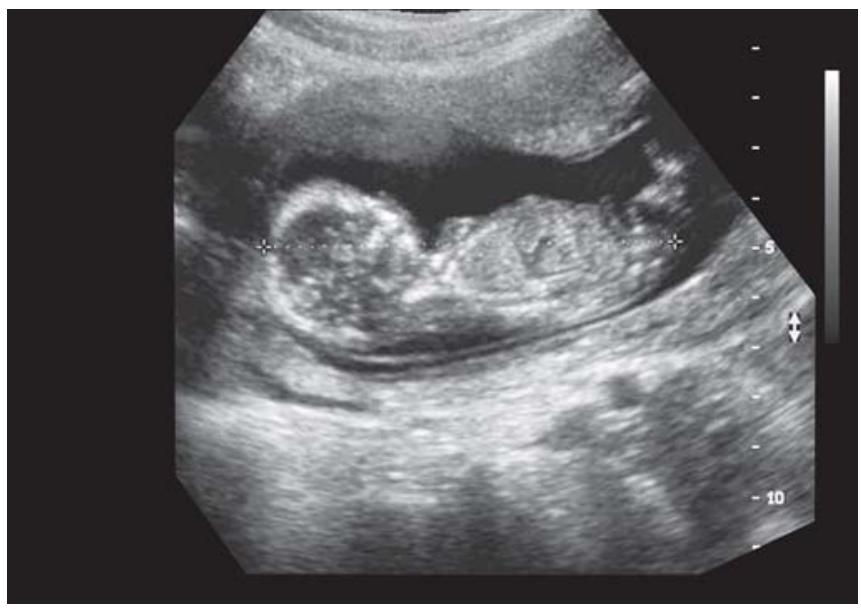

Fig. 3: Normal postoperative US with fetal heart rate monitoring 
In sporadic pheochromocytoma, symptoms can be misdiagnosed as preeclampsia, which is by far the most common cause of hypertension during pregnancy. ${ }^{6}$ Preeclampsia is characterized by hypertension, proteinuria, peripheral edema, headache and hyperreflexia; symptoms that may be difficult to differentiate from a pheochromocytoma. Preeclampsia resolves with delivery whereas symptoms related to pheochromocytoma do not and may even be exacerbated during delivery.

\section{DIAGNOSIS}

Collection of a 24-hour urine for measurement of fractionated metanephrines and catecholamines is an excellent case detection test for pheochromocytoma. Plasma levels and fractionated metanephrines have a high sensitivity for detecting pheochromocytoma but have a higher false positive rate than urinary studies. Plasma levels are used more frequently in children or in familial cases with a high index of suspicion.

MRI is the imaging method of choice in a pregnant patient because it lacks ionizing radiation. Pheochromocytomas on MRI display a high signal intensity on T2-weighted images and have high water density on chemical shift MRI, offering both anatomical and physiologic evidence of a pheochromocytoma. Our patient had a CT scan performed before her pregnancy. In nonpregnant patients, CT is the best and most cost-effective imaging modality with an overall accuracy greater than $90 \% .^{7}$ Also in nonpregnant patients, nuclear medicine studies, such as ${ }^{123}$ I-metaiodobenzylguanidine (MIBG) scintigraphy has reported sensitivities of 80 to $90 \%$ as the agent concentrates in adrenergic vesicles.

It is of critical importance to provide adequate alphaadrenergic blockade followed beta-adrenergic blockade when possible in the same fashion as nonpregnant patients. Phenoxybenzamine is safe and well tolerated during pregnancy. The desired end point of $\alpha$-adrenergic blockade is low normal blood pressure for age. Patients are encouraged to consume a high sodium diet and to increase their fluid intake to expand their intravascular volume with hopes of minimizing blood pressure swings intraoperatively and prevent hypotension postoperatively. We usually add a $\beta$-adrenergic blockade 2 to 3 days prior to surgery and titrate for a target heart rate of 80 beats per minute. There is concern with the use of beta-adrenergic blockade during pregnancy because of the potential for intrauterine growth retardation, but the advantages far outweigh these risks.

Pheochromocytoma is a life-threatening tumor and should be treated aggressively. We recommend operating during the second trimester of pregnancy as the benefits of a controlled and planned operation outweigh the risks of term delivery with a concurrent pheochromocytoma. Our operation of choice is a laparoscopic adrenalectomy. Other options include cortical sparing adrenalectomy which may obviate the need for long-term corticosteroid replacement therapy. However, there is a known increase of recurrent pheochromocytoma when the cortex is spared and up to two-thirds of patients ultimately require ongoing corticosteroid replacement. $^{8}$

If the fetus is greater than 24 weeks gestation, medical management can be attempted with very close maternal and fetal monitoring. Once the fetus is mature, cesarean section should be performed followed by tumor excision.

\section{CONCLUSION}

Pheochromocytomas are rare in pregnancy. Female patients with known familial predisposition for pheochromocytomas should have yearly case detection testing. If pregnancy is desired the recommendation is to do additional case detection testing prior to the pregnancy, including adrenaldirected imaging with CT or MRI.

In cases in which a pheochromocytoma is detected during pregnancy, early intervention via a planned laparoscopic procedure with adequate preoperative medical preparation can be achieved with high success rates. If the pheochromocytoma is discovered after the 24th week of gestation, it is not unreasonable to medically manage the patient until the fetus is viable at which point both a cesarean section and the removal of the tumor can be performed during the same operative time.

An integrated multidisciplinary approach with close communication between endocrinologist, surgeons and perinatal services is paramount to achieve excellent outcomes for both patients.

\section{REFERENCES}

1. Oliva R, Angelos P, Kaplan E, Bakris G. Hypertension. Pheochromocytoma in pregnancy: A case series and review Mar 2010;55(3):600-06.

2. Lenders J, Eisenhofer G, Mannelli M, Pacak K. Lancet. Pheochromocytyoma 20-26 Aug 2005;366(9486):665-75.

3. Neumann HP, Bausch B, McWhinney SR, et al. Germ-line mutations in nonsyndromic pheochromocytoma. N Engl J Med. 9 May 2002;346(19):1459-66.

4. Pomares FJ, Canas R, Rodriguez JM, Hernandez AM, Parrilla P, Tebar FJ. Differences between sporadic and multiple endocrine neoplasia type 2A pheochromocytoma. Clin Endocrinol 1998;48:195-200.

5. Lyman D. Paroxysmal hypertension, pheochromocytoma and pregnancy. J Am Board Fam Pract 2002;15:153-58. 
6. Grodski S, Jung C, Kertes P, et al. Phaeochromocytoma in pregnancy. Intern Med J Sep 2006;36(9):604-06.

7. Mittendorf EA, Evans DB, Lee JE, et al. Pheochromocytoma: Advances in genetics, diagnosis, localization and treatment. Hematol/Oncol Clin North Am 2007;21:509-25.

8. Yip L, Lee JE, Shapiro SE, et al. Surgical management of hereditary pheochromocytoma. J Am Coll Surg Apr 2004; 198(4):525-34, discussion 534-35.

\section{ABOUT THE AUTHORS}

\section{Benzon M Dy}

Resident, Department of General Surgery, Mayo Clinic, Minnesota United States

\section{Eitan Podgaetz}

Resident, Department of General Surgery, Mayo Clinic, Minnesota United States

\section{William F Young}

Professor, Department of Endocrinology, Mayo Clinic, Minnesota United States

\section{Geoffrey B Thompson (Corresponding Author)}

Section Head, Department of Endocrine Surgery, Mayo Clinic 200 1st Street SW, Rochester, Minnesota-55905, United States e-mail: thompson.geoffrey@mayo.edu 